\title{
Dynamic risk and reliability assessment for ship machinery decision making
}

\author{
K. Dikis, I. Lazakis, A.L. Michala, Y. Raptodimos \& G. Theotokatos \\ University of Strathclyde, Glasgow, Scotland, United Kingdom
}

\begin{abstract}
The proposed research, through INCASS (Inspection Capabilities for Enhanced Ship Safety) FP7 EU funded research project tackles the issue of predictive ship machinery inspection by enhancing reliability and safety, avoiding accidents, and protecting the environment. This paper presents the development of Machinery Risk/Reliability Analysis (MRA). The innovation of this model is the consideration and assessment of components' risk of failure and reliability degradation by utilizing raw input data. MRA takes into account the system's dynamic state change, concerning failure rate variation over time. The presented methodology involves the generation of Markov Chains integrated with the advantages of Bayesian Belief Networks (BBNs). INCASS project developed a measurement campaign, where real time sensor data is recorded onboard a tanker, bulk carrier and container ship. The gathered data is utilized for MRA DSS tool validation and testing. Following research involves components and systems interdependencies and feed the continuous dynamic probabilistic condition monitoring algorithm.
\end{abstract}

\section{INTRODUCTION}

Recent research shows that competition in maritime market develops more compound and pretentious structure affected by parameters as time, economical restraints, technology and innovation, quality, reliability and information management. In relation to successful business competence, strategic planning should be enhanced considering assets availability, involving maintenance and reliability operational aspects. The latest technology controlling these parameters is focused on monitoring the condition of main and auxiliary machinery.

The INCASS (Inspection Capabilities for Enhanced Ship Safety) FP7 EU funded research project aims to tackle the issue of ship inspection, identification of high-risk ships, providing access to information related to ship surveys and incorporate enhanced and harmonized cooperation of maritime stakeholders in order to avoid ship accidents, promote maritime safety and protect the environment.

This paper aims to present the development of a Machinery Risk and Reliability Assessment (MRA) methodology for ship machinery and equipment as well as the MRA Decision Support System (DSS). The innovation of MRA methodology is oriented towards the components' failure and state interdependencies providing a holistic view of systems' reliability performance. Furthermore, MRA takes into account the system's dynamic state change, involving failure rate variation within time. In order to approach and simulate realistically this dynamic condition monitoring control, a continuous dynamic monitoring model is introduced. The presented methodology involves the generation of Markov Chain arrangement integrated with the advantages of Bayesian Belief Networks (BBNs).
All progress and methodology development takes place using Object Oriented Programming (OOP) environment in Java language. Additionally, the MRA DSS tool is developed and introduced. This tool utilizes the MRA results by integrating historical data and expert judgment in order to assist the ship machinery inspection and maintenance. Moreover, userfriendly Graphical User Interface (GUI) is developed by involving useful DSS aspects for onboard risk and reliability control. Lastly, INCASS project developed a measurement campaign, where real time sensor data is recorded onboard a tanker, bulk carrier and container ship. The gathered data will be utilized for MRA DSS tool validation. The entire MRA DSS tool is demonstrated in this paper through a case study by employing currently simulated input data. Future research steps include the MRA DSS validation using real-time recorded data and the components' influence interdependencies.

Hence, this paper is structured in 4 sections. First of all, Section 1 introduces the paper's scope and motivation of research. Section 2 refers to the research background which involves the exploration of Condition Based Maintenance (CBM) methodology and well known Condition Monitoring (CM) technologies and tools. In Section 3 the suggested Machinery Risk Analysis (MRA) methodology is presented by demonstrating a case study, the MRA DSS and the performed results. Section 4 concludes with the discussions and future work for the MRA development.

\section{RESEARCH BACKGROUND}

This section demonstrates the latest research background with regards to maintenance control and Condition Based Maintenance (CBM) methodology. 
Moreover, this section presents the most-known failure and risk analysis methods and the latest Condition Monitoring (CM) technologies and the tools.

\subsection{Condition Based Maintenance (CBM)}

From operational viewpoint, maintenance is restructured from reactive to proactive actions, involving more control and information of the considered machinery or system (Dikis et al., 2015b). In this respect, maintenance methodologies can be identified as policies indicating the entire business's profile. These methodologies set the corporate orientation with respect to the applied maintenance strategy and operations. Different methodologies are introduced in the literature (Mobley et al., 2008). Research presents integration of methodologies and policies, allowing the utilization of flexible frameworks. CBM is the latest and under continuous development methodology. The scope of CBM is to detect the upcoming failures before even taking place, aiming to enhance machine's availability, reliability, efficiency and safety, by reducing maintenance costs through controlled spare part inventories (Mechefske, 2005). On the industrial aspect, SKF (2012) states that CBM aims at understanding of risks and predetermination of strategic actions, leading to reliability and operational cost reduction.

\subsection{Failure and risk analysis methods}

Deteriorating systems developed for the maritime industry consider internal and external to system failures as interdependencies occur during operation (Delia and Rafael, 2008). Literature presents various failure and risk analysis methods, where the majority of approaches visualize failure occurrence as independent event for each considered component of a system. These can be summarized as Fault Tree Analysis (FTA), Dynamic FTA (DFTA) taking into account time variation, Failure Mode and Effect Analysis (FMEA) and Failure Mode Effect and Criticality Analysis (FMECA), Markov Analysis (MA) and Bayes' Theorem presenting the Bayesian Belief Networks (BBNs).

On the other hand, Lazakis et al. (2010) present a predictive maintenance strategy utilizing Failure Modes, Effects and Criticality Analysis (FMECA) and Fault Tree Analysis (FTA). The model upgrades the existing ship maintenance regime to an overall strategy including technological advances and Decision Support System (DSS) by combining existing ship operational and maintenance tasks with the advances stemming from new applied techniques. On the other hand, Lazakis and Olcer (2015) introduce a novel Reliability and Criticality Based Maintenance (RCBM) strategy by utilizing a fuzzy multiple attributive group decision-making technique, which is further enhanced with the employment of Analytical Hierarchy Process
(AHP). The outcome of this study indicates that preventive maintenance is still the preferred maintenance approach by ship operators, closely followed by predictive maintenance; hence, avoiding the ship corrective maintenance framework and increasing overall ship reliability and availability. An innovative ship maintenance strategy is presented by Turan et al. (2011) based on criticality and reliability assessment while utilizing the FTA tool with time-dependent dynamic gates so as to represent in an accurate and comprehensive way the interrelation of the components of a system. As part of the reliability assessment, the Birnbaum and Criticality reliability importance measures are utilized to validate the results of the analysis, whereas a case study of a diving support vessel (DSV) illustrates the application of this strategy. In order to layout CBM and the processes that consists of; Tsang et al. (2006) suggest a data structure leading to decision analysis according to machinery's condition, proposing a method for data-driven CBM.

\subsection{Condition Monitoring (CM) technologies}

CM technology is applied through various tools. These tools record and evaluate measurable parameters that will be reviewed in this section such as vibration monitoring, acoustic and ultrasonic monitoring, thermography and oil analysis. CM is identified in phases between data acquisition, signal pre-processing and feature extraction, signal analysis and fault detection, leading to decision-making and failure prognostics (Delvecchio, 2012). This section is focused on the first phase of data acquisition. This phase involves the input data record such as displacement, velocity, acceleration, temperature, sound signal and oil analysis parameters.

Vibration monitoring is the most known technique. It offers early indication of machinery malfunctions by involving rotational speed, loading frequency, environmental conditions and material state parameters. These parameters are measured by employing different types of sensors such as; noncontact displacement transducers; velocity transducers and accelerometers (Dikis et al., 2015a). On the other hand, thermography is a tool, which is applicable to both electrical and mechanical equipment, and is deployed to identify hot and cold spots providing early signs of equipment failure. As claimed by Bagavathiappan et al. (2013), Infrared Thermography (IRT) is one of the most accepted CM tools. Due to the non-contact function is suitable for detecting structural, machinery, electrical and material malfunctions. Thermography requires thermal cameras and thermocouples for recording temperature of machinery, electrical and electronic installations. 


\subsection{Risk and reliability analysis methods}

Risk and reliability analysis methods assess various failure case scenarios of deteriorating systems and their contributing subsystems and components. Literature presents various failure and risk analysis methods, where the majority of approaches visualize failure occurrence as independent event for each considered component of a system. The analysis tools examine risk of failure by taking into account quantitative and qualitative aspects. These tools can be summarized as Event Tree Analysis (ETA), Fault Tree Analysis (FTA), Dynamic FTA (DFTA) taking into account time dependence, Failure Mode and Effect Analysis (FMEA) and Failure Mode Effect and Criticality Analysis (FMECA), Markov Analysis (MA) and Bayes' Theorem presenting the Bayesian Belief Networks (BBNs). The latter one examines the reliability performance on system, subsystem and components levels by considering functional interdependencies among them. This key feature of BBN is significant and innovative, compared to the remaining methods, as it allows the simulation of functions and operations on actual modelling environment. The $\mathrm{BBN}$ is defined as probabilistic graphical model involving conditional dependencies arranged into Directed Acyclic Graphs (DAG) and it is expressed as presented in Equation 1 (Dikis et al., 2014).

$$
P(A \mid B)=\frac{P(B \mid A) * P(A)}{P(B)}
$$

Where $\mathrm{P}(\mathrm{A})$ and $\mathrm{P}(\mathrm{B})$ are the probabilities of events $\mathrm{A}$ and $\mathrm{B}$, while $\mathrm{A}$ given $\mathrm{B}$ and $\mathrm{B}$ given $\mathrm{A}$ are conditional probabilities (* stands for multiplication). Furthermore, innovative features of BBNs involve the utilization of decision making and cost functions.

\section{SUGGESTED MRA METHODOLOGY}

In this section, the MRA methodology is demonstrated targeting to be applied on critical ship machinery and equipment of three different ship types such as tanker, bulk carrier and container ship (INCASS, 2014a). Hence, the MRA methodology is flexible in order to fulfil all requirements and specifications for each of these three ship types (INCASS, 2014b). Motivation is based on the fact that researchers' and market's tendency involves the holistic consideration of operational and failure interdependencies among multiple components within the same or different system. The MRA input data flow consists of three stages, the data acquisition and processing, the risk and reliability assessment model and the Decision Support System (DSS).

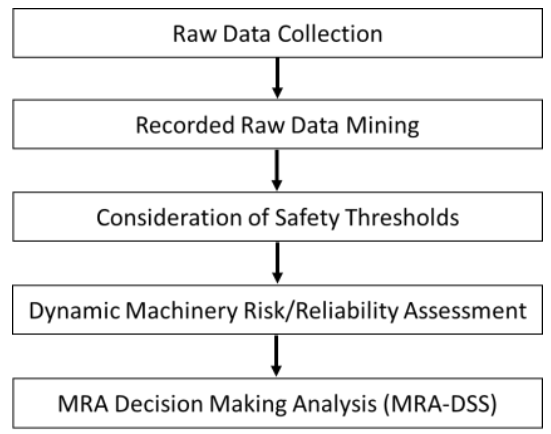

Figure 1. MRA and MRA DSS process flow.

All processing, MRA functions and DSS features are developed in Java Object Oriented Programming (OOP) language. Java is chosen as it is cross platform and allows ease of use and compatibility among different Operating Systems (OS). Figure 1 demonstrates the Machinery Risk/Reliability Analysis (MRA) methodology with respect to the process flow. On the first stage, the data acquisition and processing is considered by involving the raw data collection, mining and the safety thresholds. The input data is classified into the database on system, subsystem and component levels. The input data types are considered as historical, expert and real time monitoring data (sensor raw input). Historical input data involves past failures and records. On the other hand, expert input/judgement takes into account comments, reports and knowledge from ship crew. Real time sensor input consists of raw (unprocessed) physical measurements such as temperature and pressure. All gained information is stored in a database and transmitted in the various methodology stages utilizing 'text' (.txt) files. This format file is selected as files are small in size and can be easily and inexpensively transferred from the onboard to the onshore environment (INCASS, 2015).

The following phase involves the real monitoring data/signal processing (i.e. recorded raw data mining). Real time input data is transformed to reliability input by considering the density of abnormal measurements within the dataset as well as the amplitude of the gathered data. Abnormal levels are assessed with respect to manufacturer's acceptable operational levels. This input data type transformation from physical measurements to probabilistic indices is achieved by employing data clustering analysis of k-means (Jain and Dubes, 1988). On the other hand, literature demonstrates alternative probabilistic model approaches by utilizing mixture models such as Gaussian Mixture Model (GMM), c-means and hierarchical clustering (Theodoridis, 2015), (Jain et al., 1999), (Hand et al., 2001). The following process of the MRA methodology employs the physical measurements' thresholds. In other words, the safety indices are considered by setting the acceptable operational levels. These safety levels identify the acceptable and warning limits of the physical measurements that the system should function. Furthermore, the integration 
of the data clustering analysis with the identification of the safety thresholds introduces the probability of occurrence the observed (recorded) input data to perform within the acceptable functional levels. This probabilistic measure in percentage generates the input for the following risk and reliability tool.

The data clustering approach of k-means aims to partition the n observations into $k(\leq n)$ sets $S=\left\{S_{1}, S_{2}\right.$, ..., $\left.S_{k}\right\}$ so as to minimize the Within-Cluster Sum of Squares (WCSS) (sum of distance functions of each point in the cluster to the $\mathrm{K}$ center). A recorded dataset (raw data collection) $\left(x_{1}, x_{2}, \ldots, x_{n}\right)$ is observed, where each observation is a d-dimensional real vector. Hence, k-means data clustering model scope is to find (Theodoridis, 2015):

$$
\underset{\mathbf{S}}{\operatorname{argmin}} \sum_{i=1}^{k} \sum_{x \in S_{i}}\left\|x-\mu_{\iota}\right\|^{2}
$$

where $\mu_{i}$ is the mean of points in $S_{i}$ as shown in Equation 2.

$\mathrm{K}$-means data clustering approach is selected as it is suitable for large number of variables. $\mathrm{K}$-means is one of the simplest algorithms which uses unsupervised learning method to solve known clustering issues. Moreover, k-means can be computationally faster than hierarchical clustering methods. On the other hand, k-means can produce tighter clusters than hierarchical clustering. Additionally, k-means enables high flexibility in data analysis as it becomes a great solution for pre-clustering, reducing the space of each cluster and allowing the integration with other algorithms for further processing.

In the second stage 'Reliability Model', the processed reliability input data is introduced. The risk and reliability model employs a network arrangement similar to the Bayesian Belief Networks (BBNs). This selection allows the probabilistic modelling by considering functional relations and system, subsystem and component interdependencies. In the case of dynamic modelling, the time dependencies and state division of the reliability input are developed in parallel with the network model. The MRA application employs the mathematical tool of Markov Chains (MC) (Fort et al., 2015). MC is mathematical system that undergoes transitions from one state to another on a state space.

Furthermore, MC is selected as it is flexible to set up by allowing different levels of state sequence complexity. In order to understand the dynamic probabilistic modelling, a schematic diagram is presented in Fort et al. (2015). The presented subsystem sample includes in total three states within the timeline. Firstly, historical processed data from the previous time slice are provided shown as $t-1$. The current state $(t)$ is calculated, whereas the predictive state is shown as future state $t+1$. As it can be seen in Figure 2 each time slice $(t-1, t, t+1)$ is based on the previous state. This single state transition from past to present and then to forecasted future is known as Markov Chain (MC). The generic probabilistic expression is shown in Equation 3. On the other hand, Equation 4 presents the PoW per expressed component/subsystem in the future $t+1$ time slice. Where, $P\left(w_{t+1}\right)$ denotes the PoW in future state $(t+1)$ by taking into account previous working and failing states $P\left(w_{t}\right)$ and $P\left(f_{t}\right)$ respectively.

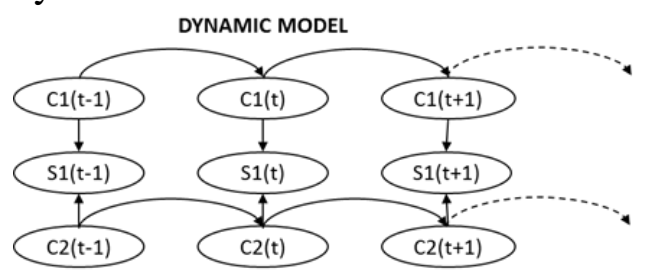

Figure 2. Dynamic probabilistic network arrangement.

$$
\begin{aligned}
& P_{X(n-1), X(n)}=P\left\{X_{t_{n}}=X_{n} \mid X_{t_{n-1}}=X_{n-1}\right\} \\
& P\left(w_{t+1}\right)=P\left(w \mid w_{t}\right) P\left(w_{t}\right)+P\left(w \mid f_{t}\right) P\left(f_{t}\right)
\end{aligned}
$$

While, each component of a sub-system is linked with a certain number of failure modes that varies between components, a generic form expressing the failure case scenarios is presented in Equation 7. In this expression, $P$ denotes the Probability of Survival (PoS) for different failure scenarios, where $w$ shows the PoW state while $f$ shows the PoF. The relation of $w$ and $f$ is shown in in Equation 8. Whereas, $f_{f t}$ indicates the failure mode (i.e. noise, vibration, overheating etc.).

Specifically, $P_{1}$ denotes the PoW and PoF states while one failure mode takes place $\left(f t_{f l}\right)$ (Equation 4). Accordingly, $P_{2}$ denotes the PoW state for a different failure mode $\left(f_{f_{2}}\right)$ (Equation 5). Whereas, $P_{3}$ represents the PoW and PoF states while $f_{f t}$ and $f_{t}$ take place at the same time (Equation 6).

$$
\begin{aligned}
& P_{1}=\left\{\begin{array}{l}
w: 100-f t_{f 1} \\
f: \quad f t_{f 1}
\end{array}\right. \\
& P_{2}=\left\{\begin{array}{l}
w: 100-f t_{f 2} \\
f: \quad f t_{f 2}
\end{array}\right. \\
& P_{3}=\left\{\begin{array}{ll}
w: 100-\left(f t_{f 1} * f t_{f 2}\right) \\
f: & \left(f t_{f 1} * f t_{f 2}\right)
\end{array} ;\right. \\
& P_{m}=\left(f t_{f 1} * f t_{f 2} * f t_{f 3} * \ldots * f t_{f k}\right) \\
& f=100-w
\end{aligned}
$$

Equation 9 shows the relation among working and failing state reliability performance. Equation 10 presents the generic expression of the overall PoS per component, including the summation of all possible break down scenarios ( $m$ : total amount of failure scenarios) and the summation of all considered failure types ( $k$ : total amount of failure types). In addition the relation of $\mathrm{m}$ and $k$ is presented in Equation 11. 


$$
P(\operatorname{comp})=\sum_{j=1}^{m}\left(\sum_{i=1}^{k} P\left(f t_{f(i)}, f t_{f(j)}\right)\right)
$$

$$
m=2^{k}
$$

The third stage of the MRA tool implements the Decision Support System (DSS) aspects. The MRA DSS methodology is divided into two sections. The first one utilizes local (onboard) and short term decision making suggestions, whereas the second one is used onshore (global) for longer term predictions and decision features. The MRA DSS demonstrates the considered systems, subsystems and components into a tree structure form. The operator has the option of choosing each of these and getting information related to past, current and predicted reliability performance. This research paper is focused on the Machinery Risk and Reliability Assessment (MRA) tool. Hence, the introduced application, in the following section, performs utilizing the MRA methods and the risk and reliability aspects.

\subsection{MRA case study}

In this section, a Machinery Risk/Reliability Analysis (MRA) case study is presented by involving the ship Main Engine $(\mathrm{M} / \mathrm{E})$, two subsystems and multiple components. The case study assesses the working state reliability performance on subsystem and component levels by analyzing various probable failure case scenarios. The case study employs simulated input data that are generated utilizing normal distribution (Gaussian). The safety thresholds (i.e. safety indices) are identified through the engine's manufacturer's manual and the engine's sea trials. These safety indices are selected as they fulfil the manufacturer's requirements and sea trials provide the ideal available reference point for further comparison.

The model's arrangement considers the ship Main Engine (M/E), the cylinder assembly and starting air subsystems. In the case of the first subsystem four components are involved such as the crosshead bearing, piston lube oil, piston liner and cylinder safety valve, whereas in the case of the starting air subsystem the cylinders' starting air valves, scavenging air receiver and scavenger air distributor are taken into account. Most of these components, where applicable, are analyzed with respect to 6 items per component as the engine's manual used is from a six-cylinder marine diesel engine. Table 1 demonstrates the raw input data requirements that MRA methodology is tested.
Table 1. Raw input data requirements.

\begin{tabular}{llll}
\hline System & Component & Measurement & Unit \\
\hline Cylinder & Crosshead Bearing & Temperature & ${ }^{\circ} \mathrm{C}$ \\
& Piston Lube Oil & Temperature & ${ }^{\circ} \mathrm{C}$ \\
& Piston Liner & Pressure & bar \\
& Cylinder Safety & Pressure & bar \\
& Valve & Temperature & ${ }^{\circ} \mathrm{C}$ \\
Start Air & Start Air Distributor & Pressure & bar \\
& Start Air Receiver & Pressure & bar \\
& Start Air Valves & Pres
\end{tabular}

Figure 3 demonstrates the Main Engine (M/E) MRA network case study. This network consists of crosshead bearings, piston lube oil measures (one per cylinder), cylinder liners and safety valves, cylinder starting air valves, scavenger air receiver and scavenger air distributor. There are two modelling approaches to structure this network. The first approach links the involved components directly to the cylinder assembly and starting air system nodes. The second approach as shown in Figure 3 takes into account an intermediate level of nodes that sums up the predictions of the working state reliability performance per group of identical components. Due to Equation 11, the first approach will involve 32 measures to be considered for the calculation of the overall cylinder assembly subsystem. This relation $\left(\mathrm{m}=2^{\mathrm{k}}\right)$, where $\mathrm{k}=32$ (components), will create almost 4.3 billion failure case scenarios to be calculated in order the overall performance of the subsystem to be examined. This network arrangement will cause further programming effort as well as increased calculation and processing time.

On the other hand, the demonstrated network arrangement involves relations where $\mathrm{k}=6$. Hence, the developed and programmed failure case scenarios per component are limited to 64 . In other words, only 324 failure case scenarios for the seven involved components have to be assessed for the introduced MRA network arrangement. The proposed network structure advances to high calculation performance and simpler code development. On the results' perspective, the first network modeling approach (without intermediate node level) is more analytical by assessing more failure case scenarios. These scenarios assess the failure of crosshead bearings at the same time with the piston lube oil, the piston liners and the valves (as Equations 5-9 show). The combination of multiple failures creates impractical low predicted working state reliability performance. On the other hand, these analytical scenarios demonstrate the sequential failure of components (interconnections). This sequential failure assessment can be introduced in the simpler and faster proposed network arrangement by introducing the functional component interdependencies. Hence, programming and calculation effort can be gained without involving unnecessary scenarios that 
their results do not demonstrate the practical functionality of the system.

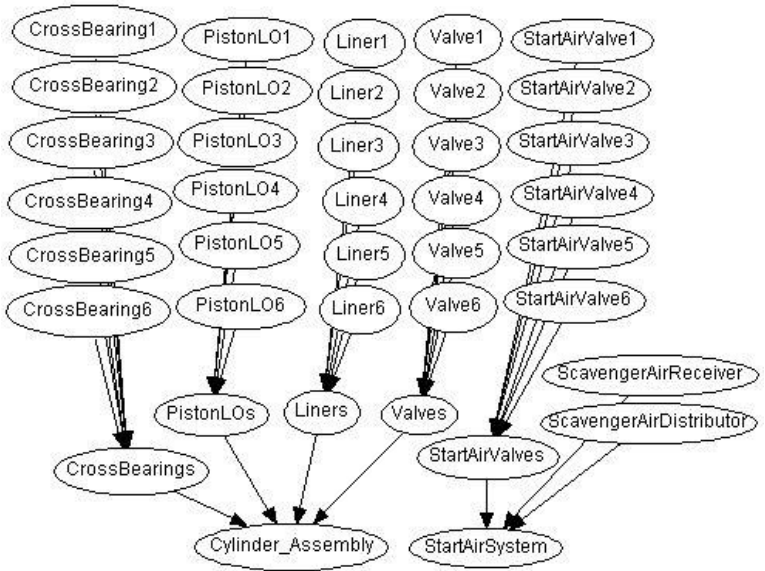

Figure 3. Main Engine (M/E) MRA network case study.

\subsection{MRA DSS case study model arrangement}

First of all, Figure 4 presents the MRA DSS analysis of failure predictions through a user-friendly Graphical User Interface (GUI). The user has available information related to cost analysis, maintenance actions, reliability performance predictions and symptoms due to reliability loss. In Figure 4 is shown the current subsystem and component reliability performance and the associated warning and failures.

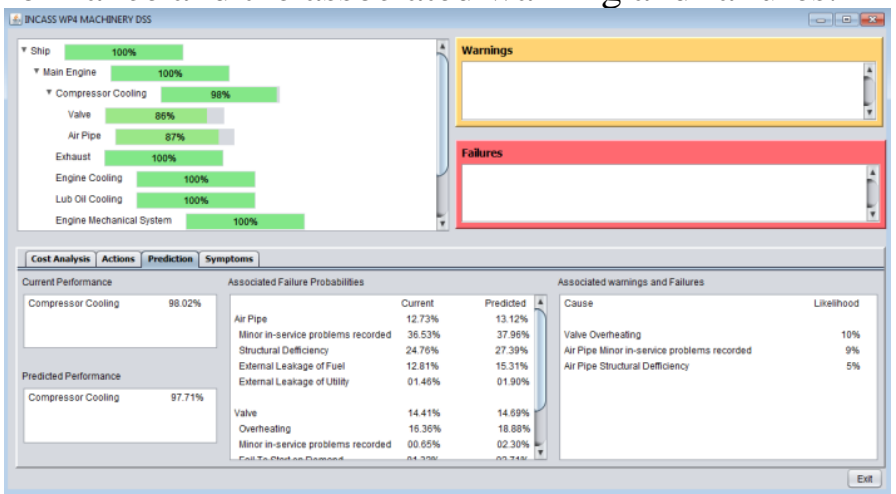

Figure 4. MRA DSS analysis of failure prediction.

On the other hand, Figure 5 demonstrates the symptoms tab in a graphical format and five days prediction in advance from the current moment. The graphs are presented in days for this occasion and with the grid marking four-hour intervals on the time axis. This is to coincide with the regular four-hourly visits the engineers onboard the ship performs.

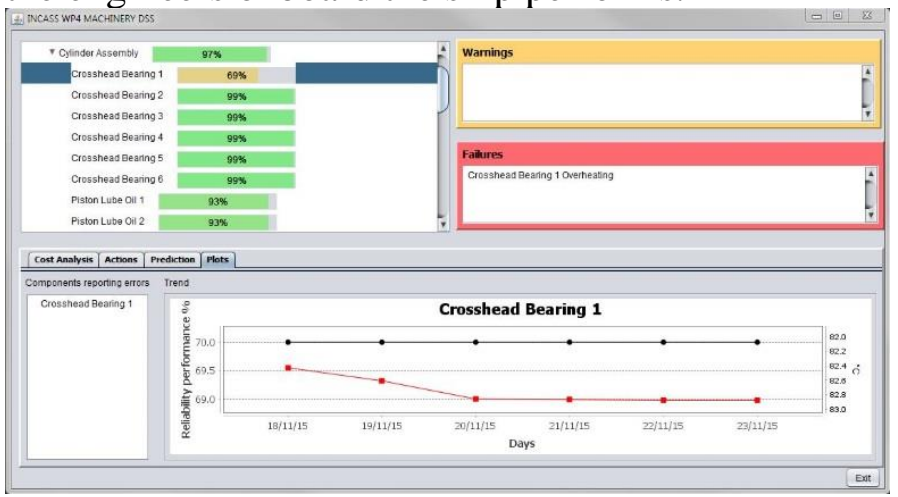

Figure 5. MRA DSS plotting of results.

\subsection{MRA case study results}

This section presents the results of the MRA Main Engine (M/E) case study. The outcomes are demonstrated on component and subsystem level. The raw input observations involve simulated datasets, 48 measurements per day and 2 days total data of historical/existing information. First of all this case study proves the ability of predicting the working state reliability performance on subsystem and component levels. This methodology introduces the requirement relation of forecasting double period of time of the provided recorded historical input. In other words, two days of existing input predicts the working state reliability performance of the following four days.

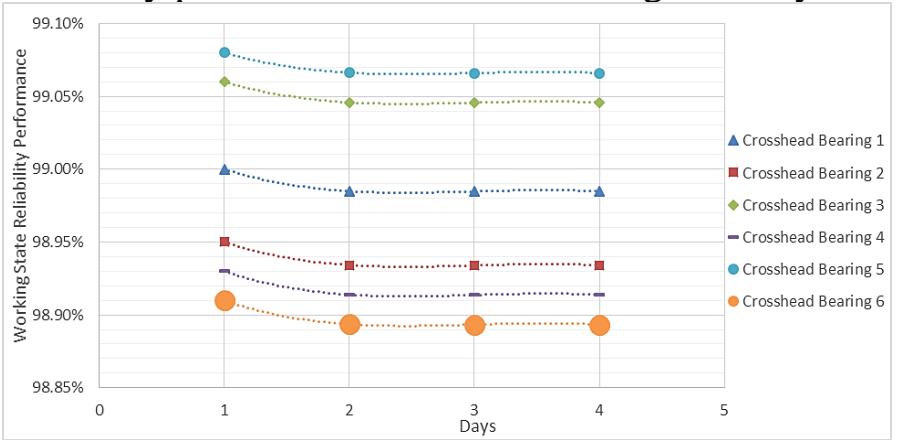

Figure 6. Reliability performance of crosshead bearings.

The figure above demonstrates the predicted working state reliability performance of the six crosshead bearings. The uniformity of the predicted results among the crosshead bearings is expected due to the utilization of simulated input datasets. Furthermore, simulating real system functioning, each component performs on different reliability levels as various parameters affect each bearing. The overall reliability performance of the crosshead bearings, as shown in Figure 6, confirms acceptable forecasted working levels. On the other hand, negligible reliability performance loss is forecasted per bearing. This minor reliability difference is expected as the employed input datasets figure only two days of performance. Hence, the upcoming forecasts perform low reliability loss for the following four predicted days. The overall reliability demonstrates performance from $99 \%$ to $98.9 \%$ and almost stable temperature at $52.3{ }^{\circ} \mathrm{C}$. The marine engine's manufacturer's manual identifies normal operational temperature levels from $50-70{ }^{\circ} \mathrm{C}$ and warning alarm level at $75{ }^{\circ} \mathrm{C}$. Hence, there is no indication of upcoming failure or abnormal component functioning.

Similarly, the six lube oil pistons' forecasted working state reliability performance are considered. Similar prediction behavior is shown as in the crosshead bearings' case. The uniform results are expected as well for the same reasoning as above. The overall working state reliability performance shows almost stable predictions at $93 \%$ (reliability) and $59.6{ }^{\circ} \mathrm{C}$. Both results are acceptable as the engine manufacturer sets the safety threshold at $65^{\circ} \mathrm{C}$ (not exceeding). 


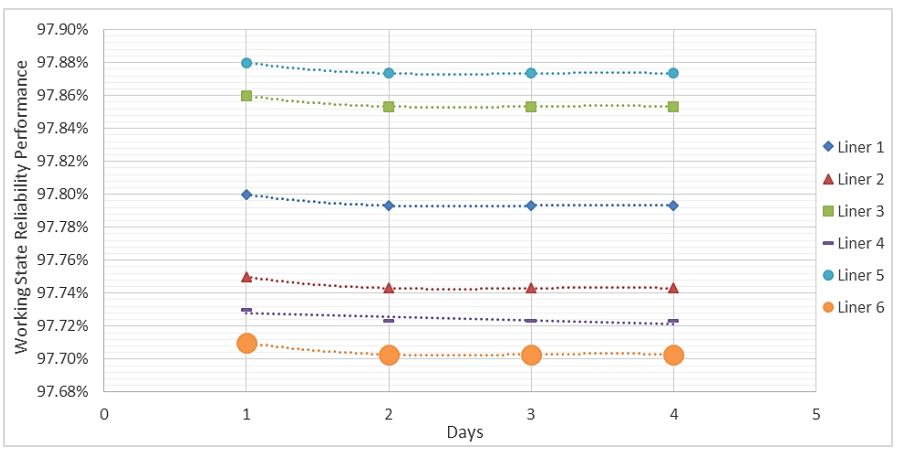

Figure 7. Reliability performance of piston liners.

In the cases of the crosshead bearings and the piston lube oil, the safety threshold sets the maximum (upper) limits that the warnings are introduced if the predictions exceed these. On the other hand, Figure 7 presents the working state reliability performance of the piston liners. This component's input measurement requires the monitoring of pressure. Hence, the manufacturer's manual utilizes safety indices that the pressure should not drop lower than the predefined levels. The overall reliability demonstrates performance at almost stably $97.8 \%$, whereas the pressure is at 132 bars. The manufacturer's requirement specifies the piston liner's pressure should not drop lower than 116 bars and should not exceed 160 bars.

Similarly, the reliability performance of the cylinder safety valves shows stable progress through time at $99.5 \%$ and 6.8 bars. In this respect, the acceptable functional level is set within the range of 6.5-7.5 bars and the warning is specified at not lower than 5.5 bars. In other words, the current reliability performance of all involved components (i.e. crosshead bearings, piston lube oil, liners and cylinder safety valves) is acceptable and there is no need for maintenance actions. As the scope of this study is to identify and examine the working state reliability performance, it is essential to highlight that the stable performance so far sets the ground for further functioning of all components.

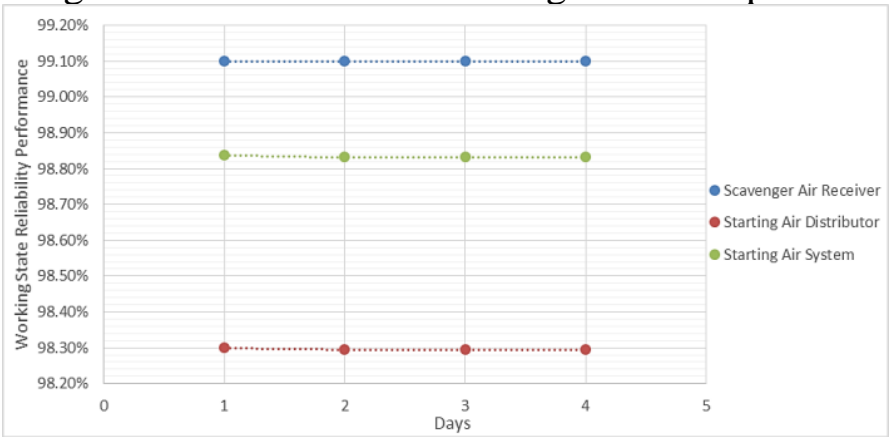

Figure 8. Reliability performance of starting air components.

Similarly, Figure 8 demonstrates the reliability performance of the scavenger air receiver, starting air distributor and starting air subsystem. The reliability levels progress stably through time higher than $98 \%$. Lastly, the overall reliability performance at subsystem level for the cylinder assembly and the starting air subsystem cases is taken place. Due to the fact, all the components perform stable reliability performance, hence the subsystem does. In the case of the subsystem reliability assessment, there is no actual measure to classify and identify a specific threshold. However, expert judgment can provide a valid indication on which level the warning should be shown and further analysis on component level can be triggered. The overall subsystem reliability performance is expected to be increased once inspection and maintenance actions are taken on component level.

\section{DISCUSSION}

This paper demonstrates the development of the Machinery Risk Analysis (MRA) tool. MRA is probabilistic risk/ reliability analysis model established through the work performed in INCASS project. The investigation of literature takes into account the human error issues and maintenance operation control that motivated this research study. Moreover, the literature review presented in this paper consists of the latest Condition Based Maintenance (CBM) methodology, the most applied and developed Condition Monitoring (CM) technologies and tools. The research is introduced by assessing the state-of-the-art of risk and reliability analysis methods.

The suggested MRA methodology is proposed as well as the MRA reliability modelling approach. The MRA methodology consists of three processing and assessment stages. The first stage involves the input data requirements, collection and processing, whereas the second stage takes into account the risk and reliability tool development. Furthermore, the third assessment stage consists of the MRA Decision Support System (DSS) and the utilization of historical, expert and predicted reliability results to assist the inspection and maintenance planning. The developed MRA methodology is focused on the risk and reliability assessment by employing various input data types such as historical, expert and real time sensor data. The methodology consists of multiple processing and assessment methods.

Firstly, the gathered datasets are analyzed by employing raw data mining process of $\mathrm{k}-$ means. This assessment identifies the tendency of the recorded input to downgrade and lead to safety threshold before it exceeds the warning level. The safety thresholds can be specified according to the identified requirements. In this case, engine manufacturer's manual is utilized providing accurate and tested reference points (i.e. sea trials) for setting the safety indices. The dynamic probabilistic network arrangement is proposed by considering flexible Markov Chains (MC) and the reliability tool based on Bayesian Belief Networks (BBNs). The proposed methodology is applied on a case study utilizing a six cylinder marine diesel engine, the cylinder assembly subsystem, and four components' measurements such as the crosshead bearings, piston lube oil, piston liners and cylinder safety 
valves. The developed MRA tool predicts the working state reliability performance on system, subsystem and component levels.

On the current research development, the dynamic risk and reliability tool is validated by ship owners, operators and service providers. According to their expert judgment, the assessed subsystem and components perform within acceptable reliability levels of ship owners', operators', service providers' and Classification Societies' requirements. On the other hand, the accuracy of the reliability tool's forecasted results is verified by employing commercial software such as Genie 2.0, Hugin 7.8 and the Markov Chain (MC) modelling using Reliability Workbench.

\section{ACKNOWLEDGEMENT}

INCASS project has received research funding from the European Union's Seventh Framework Program under grant agreement No 605200. This publication reflects only the authors' views and European Union is not liable for any use that may be made of the information contained herein.

\section{REFERENCES}

BAGAVATHIAPPAN, S., LAHIRI, B. B., SARAVANAN, T., PHILIP, J. \& JAYAKUMAR, T. 2013. Infrared thermography for condition monitoring - A review. Infrared Physics \& Technology, 60, $35-55$.

DELIA, M. C. \& RAFAEL, P. O. 2008. A maintenance model with failures and inspection following Markovian arrival processes and two repair modes. European Journal of Operational Research, 186, 694707.

DELVECCHIO, S. 2012. On the Use of Wavelet Tranform for Practical Condition Monitoring Issues. In: BALEANU, D. (ed.). Italy, Engineering Department in Ferrara: INTECH.

DIKIS, K., LAZAKIS, I., TAHERI, A. \& THEOTOKATOS, G. Risk and Reliability Analysis Tool Development for Ship Machinery Maintenance. International Symposium on "Ship Operations, Management and Economics", 28-29 May 2015 2015a Athens, Greece.

DIKIS, K., LAZAKIS, I. \& THEOTOKATOS, G. Dynamic Reliability Analysis Tool for Ship Machinery Maintenance. International Maritime Association of the Mediterranean, 21-24 September 2015 2015b Pula, Croatia. University of Strathclyde, Glasgow.

DIKIS, K., LAZAKIS, I. \& TURAN, O. Probabilistic Risk Assessment of Condition Monitoring of Marine Diesel Engines. International Conference on Maritime Technology, 7-9
July 20142014 Glasgow, UK. University of Strathclyde, Glasgow.

FORT, A., MUGNAINI, M. \& VIGNOLI, V. 2015. Hidden Markov Models approach used for life parameters estimations. Reliability Engineering \& System Safety, 136, 85-91.

HAND, D. J., MANNILA, H. \& SMYTH, P. 2001. Principles of Data Mining, MIT Press.

INCASS 2014a. Deliverable D4.1 Machinery and equipment requirement specification. INCASS - Inspection Capabilities for Enhanced Ship Safety. EC FP7 Project.

INCASS 2014b. Deliverable D4.2 Stakeholders' data requirements. INCASS - Inspection Capabilities for Enhanced Ship Safety. EC FP7 Project.

INCASS 2015. Deliverable D4.4 Machinery and equipment assessment methodology at component and system level. INCASS Inspection Capabilities for Enhanced Ship Safety. EC FP7 Project.

JAIN, A. K. \& DUBES, R. C. 1988. Algorithms for clustering data, Prentice Hall PTR.

JAIN, A. K., MURTY, M. N. \& FLYNN, P. J. 1999. Data clustering: a review. ACM Comput. Surv., 31, 264-323.

LAZAKIS, I. \& OLCER, A. I. 2015. Selection of the best maintenance approach in the maritime industry under fuzzy multiple attributive group decision-making environment. Journal of Engineering for the Maritime Environment, 13.

LAZAKIS, I., TURAN, O. \& AKSU, S. 2010. Increasing ship operational reliability through the implementation of a holistic maintenance management strategy. Ships and Offshore Structures, 5, 337-357.

MECHEFSKE, C. K. 2005. Machine Condition Monitoring and Fault Diagnosis, Boca Raton, Florida, USA, CRC Press, Taylor \& Francis Group.

MOBLEY, K., HIGGINS, L. \& WIKOFF, D. 2008. Maintenance Engineering Handbook, Mcgraw-hill.

SKF 2012. Condition-based maintenance must be set up correctly. Marine Propulsion - Ship lifecyle management.

THEODORIDIS, S. 2015. Machiner Learning: A Bayesian and Optimization Perspective.

TSANG, A. H. C., YEUNG, W. K., JARDINE, A. K. S. \& LEUNG, B. P. K. 2006. Data management for CBM optimization. Journal of Quality in Maintenance Engineering, 12, 37-51.

TURAN, O., LAZAKIS, I., JUDAH, S. \& INCECIK, A. 2011. Investigating the reliability and criticality of the maintenance characteristics of a diving support vessel. Quality and Reliability Engineering International, 27, 931-946. 